\title{
A design study on complexity reduced multipath mitigation
}

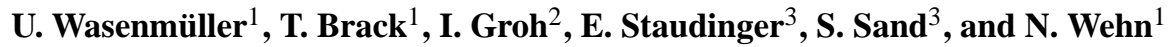 \\ ${ }^{1}$ Microelectronic Systems Design Research Group, University of Kaiserslautern, Germany \\ ${ }^{2}$ Intel Mobile Communications, Germany \\ ${ }^{3}$ Institute of Communications and Navigation, German Aerospace Center (DLR), Germany \\ Correspondence to: U. Wasenmüller (wasenmueller@eit.uni-kl.de)
}

\begin{abstract}
Global navigation satellite systems, e.g. the current GPS and the future European Galileo system, are frequently used in car navigation systems or smart phones to determine the position of a user. The calculation of the mobile position is based on the signal propagation times between the satellites and the mobile terminal. Further, the satellites need to be line-of-sight to the receiver for exact position calculation. However, in an urban area, the direct path may be blocked and the resulting multipath propagation causes errors in the order of tens of meters for each measurement.

In this paper an advanced algorithm for multipath mitigation known as CRMM is presented. CRMM features reduced algorithmic complexity and superior performance in comparison with other state of the art multipath mitigation algorithms. Simulation results demonstrate the significant improvements in position calculation in environments with severe multipath propagation. Nevertheless, in relation to traditional algorithms an increased effort is required for real-time signal processing due to the large amount of data, which has to be processed in parallel. Based on CRMM, we performed a comprehensive design study including a design space exploration for the tracking unit hardware part, and prototype implementation for hardware complexity estimation.
\end{abstract}

\section{Introduction}

Currently, personal navigation devices (PNDs) and smartphones are widely used for car and pedestrian navigation or location based services (LBS). These devices employ mass market global navigation satellite systems (GNSSs) receivers, e.g. for the current GPS and GLONASS or the future European Galileo system. A GNSS receiver determines its position uniquely by measuring and calculating the signal propagation time from at least four different satellite signals. State-of-the-art mass market GNSS receivers measure and track the time of arrival (TOA) of the satellite signals with narrow early-minus-late (NEML) delay-locked loops (DLLs). NEML DLLs result in the optimum TOA estimates when there is a direct line-of-sight (LOS) between the satellite and GNSS receiver and the signal reception is not disturbed by multipath propagation.

Often, LBS are used in cities where the satellite signals are subject to diffraction, refraction, and scattering resulting in multipath propagation. Thus, the positioning accuracy of state-of-the art mass market GNSS receivers with NEML DLLs will be degraded from a few meters to several tens of meters. In van Nee et al. (1994), maximum likelihood (ML) multipath estimation (ME) algorithms can mitigate these errors but are computationally too expensive for mass market GNSS receivers in terms of computing power and power consumption. Consequently, Selva $(2004,2005)$ developed complexity reduced multipath mitigation (CRMM) algorithms that employ a bank of correlators to reduce the amount of data that needs to be handled by the MLME. Several publications on CRMM discuss various implementation aspects of the algorithm, e.g. Groh and Sand (2008); Groh et al. (2011); Groh and Sand (2012). However, none provides a more detailed analysis with respect to real-time or hardware implementation issues.

In Sect. 2 we show the used system model and in Sect. 3 the considered CRMM algorithm is summarized. In Sect.4 the design study including design space exploration for the tracking unit hardware part and prototype implementation for hardware complexity estimation is presented, and in Sect. 5 the results are summarized.

\section{System model and MLME}

In this section, we describe briefly the underlying system model of transmitting and receiving a spread spectrum navigation signal and the MLME problem. We consider a rectangular pulse $g(t)$ with cut-off frequency $f_{N}$. The direct 


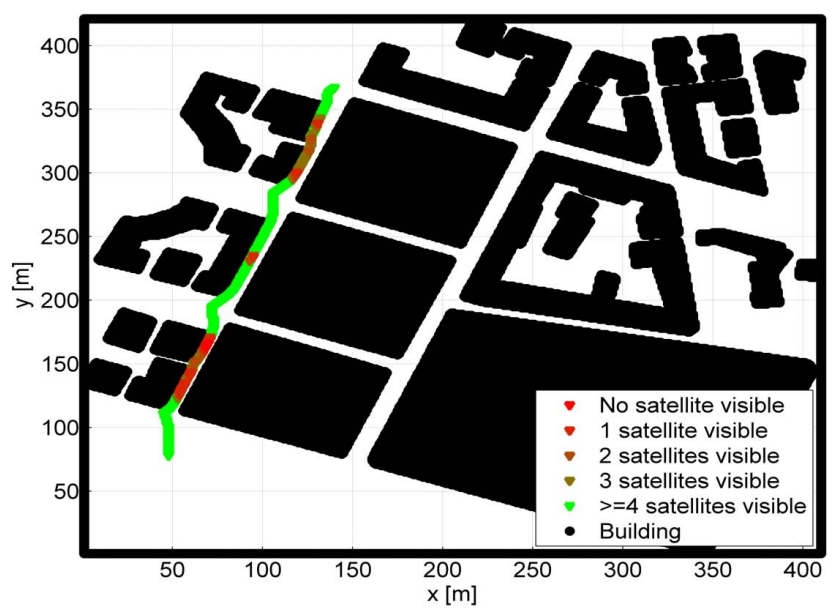

Fig. 1. GNSS satellite visibility in a Munich urban environment.

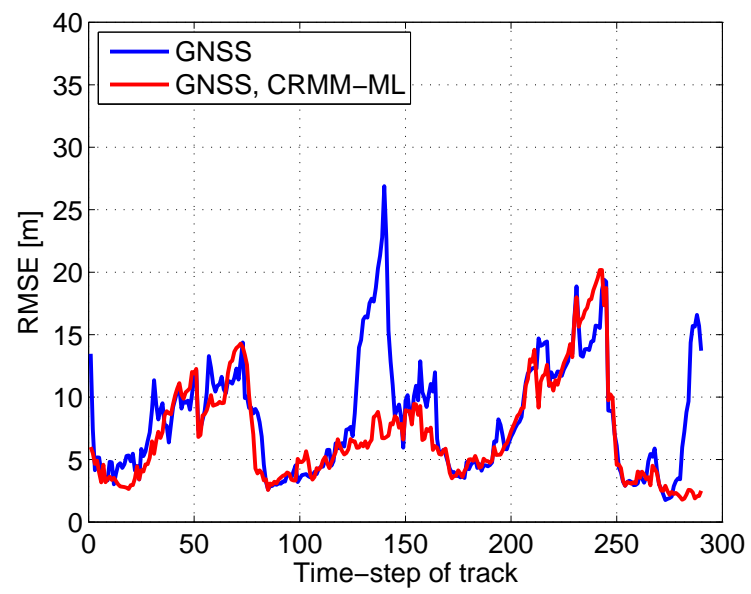

Fig. 2. RMSE vs. time-step of track for GNSS with NEML and CRMM-ML.

sequence spread spectrum transmit signal is then

$$
\begin{aligned}
s(t) & =\sum_{m=0}^{M-1} d_{\mathrm{P}, m} \sum_{n=0}^{N-1} c_{\mathrm{P}, n} g\left(t-m T-n T_{\mathrm{C}}\right), \\
& +\sum_{m=0}^{M-1} d_{\mathrm{D}, m} \sum_{n=0}^{N-1} c_{\mathrm{D}, n} g\left(t-m T-n T_{\mathrm{C}}\right)
\end{aligned}
$$

where $T=N T_{\mathrm{C}}$ and $T_{\mathrm{C}}=1 / R_{\mathrm{C}}$ denote the spreading code duration and chip duration with $R_{\mathrm{C}}$ being the chip rate. The pilot and data symbol sequences $d_{\mathrm{P}, m}$ and $d_{\mathrm{D}, m}, m=$ $0, \ldots, M-1$ are taken from quartenary phase shift keying modulation. $M$ codewords form the considered time interval for MLME. The spreading code sequences $c_{\mathrm{P}, n}$ and $c_{\mathrm{D}, n}, n=0, \ldots, N-1$ for the pilots and data are pseudo-noise sequences of length $N$ as defined in EUG (2010). In order to obtain a suitable matrix vector factorization beginning from Eq. (1), we choose the sampling frequency $f_{\mathrm{S}}$ as integer mul-

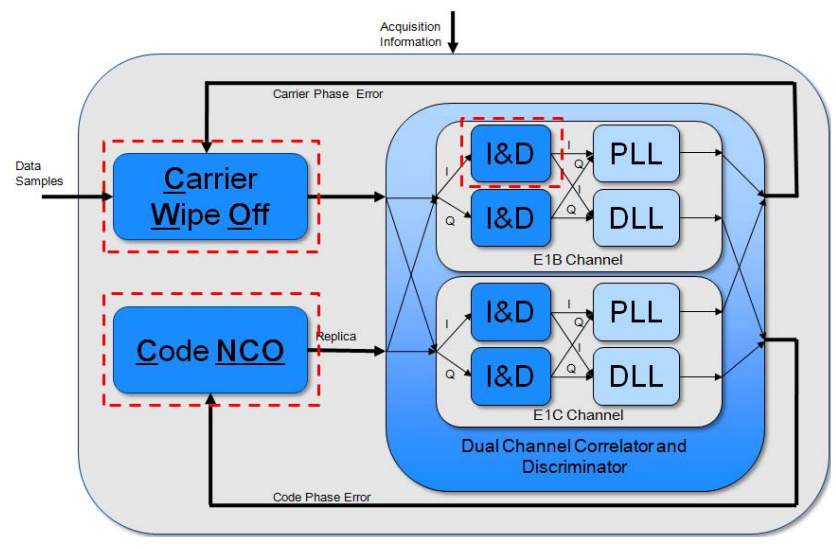

Fig. 3. Arcitecture of Tracking unit.

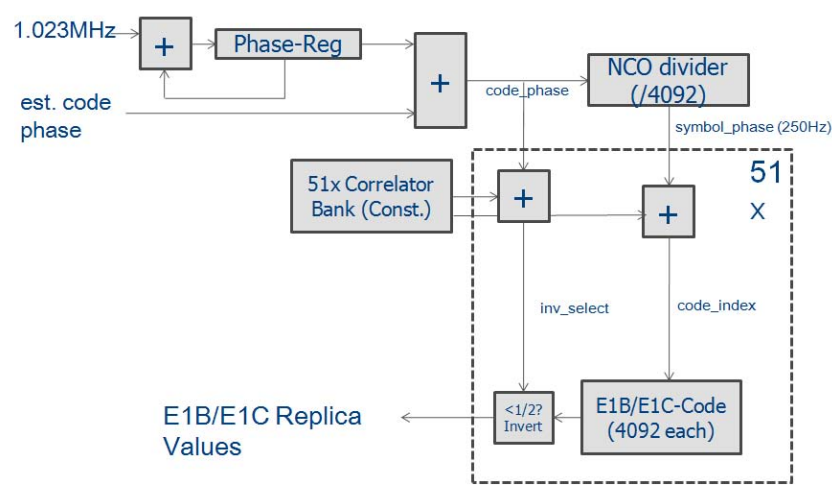

Fig. 4. Arcitecture of Code NCO and PRN unit.

tiple of the chip rate $R_{\mathrm{C}}$ yielding $Q=f_{\mathrm{S}} / R_{\mathrm{C}}$ samples per chip.

In order to demonstrate the influence of the multipath propagation on the estimation performance of the LOS path and thereby on the position estimation of the mobile station, the channel is modelled as a fixed channel of length $L$

$h(\tau, t)=h(\tau)=\sum_{\ell=1}^{L} a_{\ell} \delta\left(\tau-\tau_{\ell}\right)$

where the number of channel coefficients $L$ is assumed to be known to the receiver. $a_{\ell}$ and $\tau_{\ell}$ are the amplitude and delay of the $\ell$-th multipath component. Note that $l=1$ corresponds to the LOS component. The TOA $\tau_{1}$ is related to the distance between the $i$-th satellite and the receiver as $d_{i}=c \tau_{1}$ with $c$ being the speed of light.

After transmission over the channel (cf. Eq. 2), we obtain the complex valued baseband-equivalent received signal as

$y(t)=\sum_{l=1}^{L} a_{l} s\left(t-\tau_{l}\right)+n(t)$

where $s(t)$ is the navigation signal transmitted by the satellite and $n(t) \sim \mathcal{N}_{\mathrm{c}}\left(0, \sigma_{n}^{2}\right)$ describes the zero-mean additive white 
Table 1. Detailed assumptions and platform properties.

\begin{tabular}{lr}
\hline Description & Parameter \\
\hline Type of FPGA & Stratix II EP2S180F1020C3 \\
ALUTs & 143520 \\
Dedicated Logic Registers & 143520 \\
DSP Block Resources & 96 \\
Block Memories & $930 \mathrm{M} 512,768 \mathrm{M} 4 \mathrm{~K}$ \\
Processing clock speed (fclk) & $100 \mathrm{MHz}$ \\
Sample Clock (fs) & $16.8 \mathrm{Msps}$ (Complex Data) \\
ADC Output Quantisation & 3 bit \\
\hline
\end{tabular}

Table 2. CWO complexity estimation.

\begin{tabular}{lrrrr}
\hline Element & Parameter & Nr. & ALUT & DREG \\
\hline SIN/COS-LUT & $8 \times 6$ bit & 1 & 6 & 6 \\
Multiplier & $3 \times 3$ bit & 2 & 12 & 6 \\
Phase Accumulator & 32 bit & 1 & 0 & 32 \\
Phase Adder & 32 bit & 1 & 32 & 0 \\
Phase Error Adder & 32 bit & 1 & 32 & 32 \\
\hline Overall & & & 82 & 76 \\
\hline
\end{tabular}

Gaussian noise (AWGN) of the power $\sigma_{n}^{2}=E\left[\left|n(t)^{2}\right|\right]$. Sampling $y(t)$, we obtain the receive signal vector

$\boldsymbol{y}=\sum_{\ell=1}^{L} a_{\ell} \boldsymbol{s}\left(\tau_{\ell}\right)+\boldsymbol{n}=\mathbf{S}(\boldsymbol{\tau}) \boldsymbol{a}+\boldsymbol{n} \in \mathbb{C}^{M N Q}$,

where $\quad \mathbf{S}(\boldsymbol{\tau})=\left[\boldsymbol{s}\left(\tau_{1}\right), \ldots, \boldsymbol{s}\left(\tau_{L}\right)\right] \in \mathbb{C}^{M N Q \times L} \quad$ and $\boldsymbol{a}=\left[a_{1}, \ldots, a_{L}\right]^{\mathrm{T}} \in \mathbb{C}^{L}$ form the signal matrix and the amplitude vector. The SNR $\gamma$ is defined according to Eq. (4) as $\gamma=\|\boldsymbol{S}(\boldsymbol{\tau}) \boldsymbol{a}\|_{2}^{2} /\left(M N Q \sigma_{n}^{2}\right)$.

The MLME $\{\hat{\mathbf{a}}, \hat{\tau}\} \in \mathbb{C}^{L}$ is given according to Lentmaier and Krach (2006) by

$$
\{\hat{\boldsymbol{a}}, \hat{\boldsymbol{\tau}}\}=\underset{\{\boldsymbol{a}, \boldsymbol{\tau}\}}{\operatorname{argmin}}\|\boldsymbol{y}-\mathbf{S}(\boldsymbol{\tau}) \boldsymbol{a}\|_{2}^{2},
$$

where the first element $\hat{\tau}_{1}$ in $\hat{\boldsymbol{\tau}}$ denotes the TOA ML estimate. The received signal vector $\boldsymbol{y}$ may contain $M=10$ symbols and $N=4092$ chips per symbol with $Q=16$ samples per chip. Thus, the optimization algorithm for Eq. (5) has to process more than 650000 samples received within $40 \mathrm{~ms}$, which is prohibitive in terms of computational complexity and power consumption for a mass market GNSS receiver.

\section{CRMM}

To overcome the computational complexity of Eq. (5), Selva (2004, 2005) developed the basic CRMM algorithm, which implements the following two steps:

- Data size reduction: the large received signal vector $\boldsymbol{y}$ is transformed by a bank of correlators into a much
Table 3. CWO synthesis results.

\begin{tabular}{lrrr}
\hline Synthesis Alternative & ALUT & DREG & DSP \\
\hline Default Options & 83 & 51 & 0 \\
Forced to use DSP & 69 & 39 & 2 \\
\hline
\end{tabular}

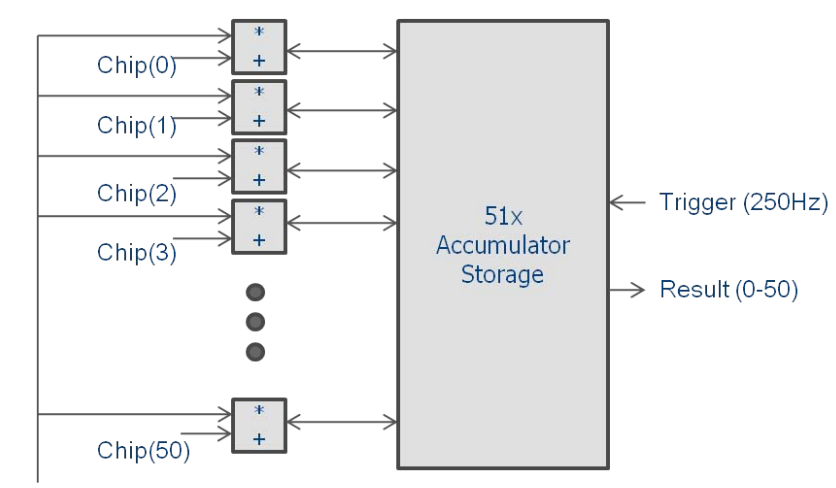

Data Samples

@16.8MSps

Fig. 5. Integrate and Dump unit.

smaller vector $\boldsymbol{y}_{\mathrm{c}}$ before the ML optimization. The subspace transform results in maximum data compression and negligible performance losses. For instance, the observation vector $\boldsymbol{y}$ can be reduced from hundred thousands of samples to $\boldsymbol{y}_{\mathrm{c}}$ with a few tens of samples Groh and Sand (2008). $\boldsymbol{y}$ can be compressed with code matched correlators (CMCs), signal matched correlators (SMCs) and principal components (PCs) or through combining either CMCs or SMCs with subsequent PCs (cf. Lentmaier and Krach, 2006; Groh and Sand, 2008, 2012). Note, in this paper we only focus on SMCs.

- ML optimization: efficient and robust Newton-type optimization algorithms were developed. These algorithms employ interpolation methods to allow arbitrary delay resolution independent of the sampling rate.

Modifications of the CRMM include extension of data size reduction to time-variant signals (Groh et al., 2011), optimized correlator computation (Groh and Sand, 2012), and replacement of the Newton-type optimization with expectation maximization or space alternating general expectation maximization algorithms (Groh and Sand, 2008).

In this paper, the focus is on the data size reduction of CRMM and its realizability in hardware in the subsequent sections. Whereas the correlator input $\boldsymbol{y}$ receives samples with rate $f_{\mathrm{S}}$, the correlator can output the samples of $\boldsymbol{y}_{\mathrm{c}}$ with rates between $1000 \mathrm{~Hz}$ to $1 \mathrm{~Hz}$ depending on $M$ and $N$. Thus, clearly subsequent complexity reduction or ML optimization will be less critical for hardware implementation.

To asses the performance of MLME with CRMM, we consider the user track depicted in Fig. 1, which shows an urban 
Table 4. NCO and PRN generator complexity estimation.

\begin{tabular}{lrrrr}
\hline Element & Parameter & Nr. & ALUT & DREG \\
\hline NCO-Divider & 32 bit by 12 bit & 1 & 48 & 48 \\
Correlator Banks & $51 \times 32$ bit & 1 & 25 & 32 \\
Phase Accumulator & $32 \mathrm{bit}$ & 1 & 0 & 32 \\
Phase Adder & $32 \mathrm{bit}$ & 1 & 32 & 0 \\
Phase Error Adder & $32 \mathrm{bit}$ & 1 & 32 & 32 \\
Correlator Adder (large) & $51 \times 32 \mathrm{bit}$ & 1 & 800 & 0 \\
Correlator Adder (small) & $51 \times 12 \mathrm{bit}$ & 1 & 36 & 0 \\
E1B/E1C Code Storage & $4092 \times 2 \mathrm{bit}$ & 3 & 480 & 0 \\
Output Registers & $2 \times 1 \mathrm{bit}$ & 51 & 0 & 102 \\
\hline Overall & & & 1453 & 246 \\
\hline
\end{tabular}

Table 5. NCO and PRN generator synthesis results.

\begin{tabular}{lcc}
\hline Synthesis Alternative & ALUT & DREG \\
\hline Default Options & 1172 & 213 \\
\hline
\end{tabular}

canyon scenario in Munich with average building height of $26 \mathrm{~m}$. For at least four LOS satellites, the track is colored green, which is the minimum number of LOS satellites to obtain a reliable position.

Figure 2 shows the corresponding root-mean-square error (RMSE) of the position estimate in meter versus the timestep of the track in Fig. 1. The RMSE was averaged over the same track with the same satellite constellation and urban scenario for 1000 noise realizations. Comparing Figs. 1 and 2, the main error source for the time-steps 20 to 85 and 180 to 255 is due to less than four available LOS satellite signals. On the other hand, the major error source at the start, for time-steps 125 to 145 , and at the end is due to multipath propagation although there should be at least four LOS satellite signals available. At these time-steps, CRMM can reduce the RMSE absolute by $7.5 \mathrm{~m}$ to $20 \mathrm{~m}$ and relative by $200 \%$ to $650 \%$.

\section{Prototype architecture design exploration}

In the GREAT project, we investigated in detail through simulations the performance of CRMM, which is superior to most simple correlators based multipath mitigation algorithms (Hu et al., 2008). CRMM requires a large number of correlators per satellite to transform the multipath mitigation into a lower dimensional sub-space. A first complexity analysis in GREAT showed that based on the floating point operations per second, CRMM has a comparable complexity with classical correlators based multi-path mitigation algorithms (Hu et al., 2008). From GREAT, the question remained open, whether it would be feasible to implement CRMM in hardware for instance in a COTS FPGA. The following study ad-
Table 6. I\&D unit complexity estimation.

\begin{tabular}{|c|c|c|c|c|c|c|c|c|c|c|}
\hline \multicolumn{5}{|c|}{ Element } & \multicolumn{2}{|c|}{ Parameter } & r. & \multicolumn{2}{|c|}{ ALUT } & DREG \\
\hline \multicolumn{5}{|c|}{ Adder } & \multicolumn{2}{|c|}{23 bit } & 1 & \multicolumn{2}{|c|}{1173} & 0 \\
\hline \multicolumn{4}{|c|}{ Accumulator Storage } & \multicolumn{3}{|c|}{$51 \times 23$ bit } & 2 & \multicolumn{2}{|r|}{0} & 2346 \\
\hline \multicolumn{8}{|c|}{ Overall } & \multicolumn{2}{|c|}{1173} & 2346 \\
\hline \multicolumn{5}{|c|}{ In: 51 Taps after $1 / 16.8 M H z$ ( $\sim 59 \mathrm{nn}), 1$. RX-Value } & \multicolumn{6}{|c|}{ In: 51 Taps after1/16.8MHz ( $\sim 59$ ns), 67200. RX-value } \\
\hline $\begin{array}{l}\text { Input } \\
1-11\end{array}$ & $\begin{array}{l}\text { Input } \\
12-23\end{array}$ & $\begin{array}{l}\text { Input } \\
\text { 24-35 }\end{array}$ & $\begin{array}{l}\text { Input } \\
36-47\end{array}$ & $\begin{array}{l}\text { Input } \\
48-51\end{array}$ & & $\begin{array}{l}\text { nput } \\
2-23\end{array}$ & $\begin{array}{l}\text { Input } \\
24-35\end{array}$ & $\begin{array}{l}\text { Input } \\
36-47\end{array}$ & $\begin{array}{l}\text { Input } \\
48-51\end{array}$ & \\
\hline \multirow[t]{2}{*}{$10 \mathrm{~ns}$} & $10 \mathrm{~ns}$ & $10 \mathrm{~ns}$ & $10 \mathrm{~ns}$ & $10 \mathrm{~ns}$ & & Ons & $10 \mathrm{~ns}$ & 10 s & 10 ns & \\
\hline & $\begin{array}{l}\text { Result } \\
1-11\end{array}$ & $\begin{array}{l}\text { Result } \\
12-23\end{array}$ & $\begin{array}{l}\text { Result } \\
24-35\end{array}$ & $\begin{array}{l}\text { Result } \\
36-47\end{array}$ & \begin{tabular}{|c} 
Reshlit \\
$48-91$ \\
1 \\
\end{tabular} & $\begin{array}{l}\text { Result } \\
1-11\end{array}$ & $\begin{array}{l}\text { Result } \\
12-23\end{array}$ & $\begin{array}{l}\text { Result } \\
24-35\end{array}$ & $\begin{array}{l}\text { Result } \\
36-47\end{array}$ & $\begin{array}{l}\text { Result } \\
48-51\end{array}$ \\
\hline
\end{tabular}

Fig. 6. Timing for alternative architecture of I\&D unit.

dresses this question in more detail with the requirement of 51 integrate and dump units.

\subsection{Design drivers and top level architecture}

Besides the acquisition unit, the tracking unit of GNSS receivers is by far the most complex receiver part which requires high-speed processing on dedicated hardware resources. Primary goal of the prototyping approach was to derive a preliminary architecture design for feasibility study and to acquire complexity estimation data for the tracking unit based on a given FPGA target platform. Detailed assumptions and platform properties are listed in Table 1.

The algorithms and internal quantisation used for the prototyping relies on the freely available GNSS dual-receiver MATLAB SIMULINK models and the corresponding "C"sources. The top level architecture of the GNSS receiver tracking unit is shown in Fig. 3, consisting of the three major units "Carrier Wipe-Off" (CWO), "Code NCO and PRN 
Table 7. I\&D unit complexity estimation (curled-up).

\begin{tabular}{lrrrr}
\hline Element & Parameter & Nr. & ALUT & DREG \\
\hline Adder & 23 bit & 11 & 253 & 0 \\
Accumulator Storage & $51 \times 23$ bit & 2 & 0 & 2346 \\
Multiplexer & $11: 1$ & $5 \times 23$ & 805 & 0 \\
De-Multiplexer & $11: 1$ & $5 \times 23$ & 805 & 0 \\
Overall & & & 1863 & 2346 \\
\hline
\end{tabular}

generator", and the "Dual Channel Correlation and Discriminators".

Each architecture unit is considered separately in the next sections. Residual elements depicted in the top level architecture (Multipliers, Registers), glue logic, and the main FSM is considered in the final complexity estimation with an adequate lump-sum estimate.

\subsection{Carrier Wipe-Off (CWO)}

The CWO unit is responsible for removing the carrier of the ADC input signal by mixing this signal with a synthetically generated carrier using the estimated frequency and phase from the acquisition and tracking components. Based on the assumptions of a 3-bit ADC input at 16.8 Msps, a 6-bit output signal with the same rate has to be generated by this unit. The basic architecture derived from the available SIMULINK model uses a so called Direct Digital Synthesizer (DDS) to generate the required complex continuous wave signal, mixing is done by per-component multiplication of the complex input signal with the generated signal. The DDS uses a phase accumulator and a sine/cosine Lookup-Table (LUT) for signal generation: A 32-bit phase accumulator was employed to achieve a frequency resolution of $0.023 \mathrm{~Hz}$ which is adequate for the used algorithm, the LUT resolution was set to 3 bit to be compatible with the input resolution. In a first step, the hardware complexity of the individual architecture components was calculated to allow for plausibility checks after synthesis. Note that the control FSM for the unit was not considered in this calculation.

In a second step, the architecture was realized in VHDL and synthesized in the Quartus-II tool suite for the appropriate FPGA target. As an alternative, the synthesis tool was forced to apply DSP blocks for the design.

Both alternatives result in a very small core requiring $<$ $1 \%$ of the full FPGA resources. To further reduce complexity, the architecture could be "curled up" to process only one sample every 5 clock cycles with the assumed clock speed of $100 \mathrm{MHz}$. This two-step approach with preliminary calculation based on architecture components followed by implementation and synthesis is also used for the remaining units.

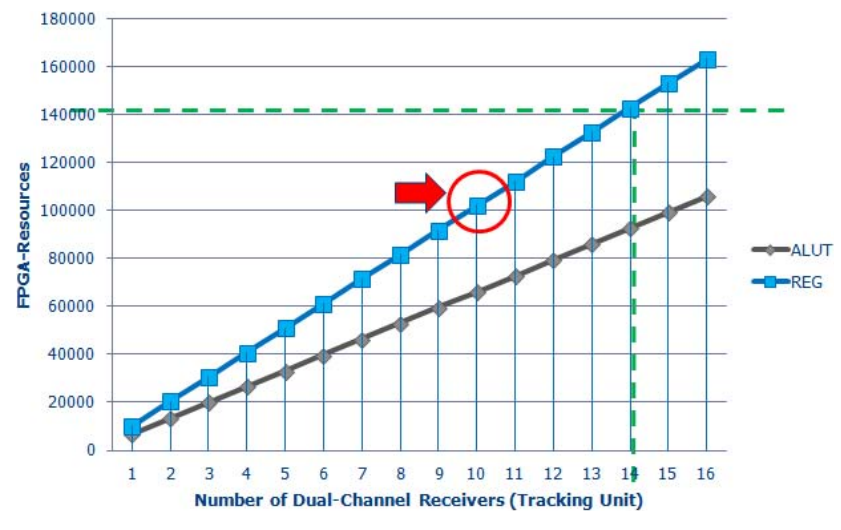

Fig. 7. Final results.

\subsection{Code NCO and PRN generator unit}

The Code NCO and PRN generator unit is utilized to generate a chip-sequence based on the given chip-rate for $1.023 \mathrm{MHz}$, the estimated code phase and the channel codes and correlators themselves. This unit accepts 32 bit codephase inputs in $4 \mathrm{~ms}$ intervals, yielding an input data rate of only $250 \mathrm{~Hz}$. The outputs of the generator are 51bit code words for both E1B and E1C channels (so called replica values) at 16.8 Mcodes per second.

The architecture uses a DDS as introduced in the CWO unit to generate code phases at the chip rate of $1.023 \mathrm{MHz}$ with an adjustable phase from the phase estimation. The frequency resolution is also set to $0.023 \mathrm{~Hz}$, so a 32bit quantisation is derived for the phase input and accumulator.

The hardware complexity for the architecture building blocks are estimated and calculated accounting for the symmetry and actual values of the provided correlators and the resulting code index values. It is determined that instead of 51 different code index values, only three actually occur given these parameters. All these properties result in the very compact Correlators Bank, Correlation Adders, and Code Storage, cf. Table 4. The synthesis results for the VHDL implementation confirm the preliminary complexity results and even undercut the estimation, cf. Table 5.

The core resembling the Code NCO and PRN generator unit utilizes $<1 \%$ of the full FPGA resources. Same as for the CWO unit, the architecture could be "curled up" to exploit the difference between sample rate and processing speed and further reduce the complexity. It is noteworthy that for different correlators and codes, the resulting complexity could be more than 10 times higher.

\subsection{Dual Channel Correlation and Discriminators unit}

The Dual Channel Correlation and Discriminators unit consists mainly of two independent channel processors which in turn contain two Integrate and Dump units (I\&D), a PLL, and a DLL each. The PLL and DLL are only triggered with a $4 \mathrm{~ms}(=250 \mathrm{~Hz})$ interval which allows for either a highly 
Table 8. I\&D unit complexity estimation (RAM-based).

\begin{tabular}{lrrrrr}
\hline Element & Parameter & Nr. & ALUT & DREG & MEM \\
\hline Adder & 23 bit & 11 & 253 & 0 & 0 \\
Adder Registers & 23 bit & 11 & 0 & 253 & 0 \\
Accumulator Storage & $8 \times 23$ bit & $11 \times 2$ & 0 & 0 & 2530 bit \\
\hline Overall & & & 253 & 253 & 2530 bit \\
\hline
\end{tabular}

Table 9. I\&D unit synthesis results (4 units).

\begin{tabular}{lrrrr}
\hline Synthesis Alternative & ALUT & DREG & M512 & M4K \\
\hline Default Options & 4692 & 9384 & 0 & 0 \\
Curled-Up & 7452 & 9384 & 0 & 0 \\
RAM-based (Auto-Fit) & 1068 & 1068 & 4 & 60 \\
RAM-based (Forced to use M512) & 1068 & 1068 & 120 & 0 \\
\hline
\end{tabular}

curled up realization or even the utilization of a general purpose processor like the NIOS-II. Using such a processor, up to 400000 clock cycles are available to achieve one PLL or DLL result. Alternatively, multiple dual-channel GNSS receivers could share one PLL/DLL processor resource. For all these reasons, the prototyping approach only considers the four I\&D units inside the Dual Channel Correlation and Discriminators unit. These units require dedicated hardware resources because of their relatively high input data rate of 16.8 MSps and large-volume data storage. Each I\&D unit has to accept the 6bit receiver data coming from the CWO unit at 16.8 Msps (I or Q component) and the 51bit wide code words coming from the Code NCO and PRN generator unit at the same rate. The I\&D unit basically multiply-accumulates the incoming data streams 67200 times which is derived from the ratio between the $16.8 \mathrm{Msps}$ sampling rate and the 250 symbol rate which also constitutes the output rate. Therefore, for each accumulator storage word 23bit are required $(6$ bit $+\log 2(67200)>22$ bit $)$. This task can be fulfilled by straight forward architecture shown in Fig. 5.

The complexity of the building blocks can be calculated from the two main building blocks of this approach, the adders and the accumulator storage itself (Table 6). To adapt for the very slow output data rate, a second "shadow storage" will be mandatory. This shadow storage keeps the last accumulation result until it can be transferred to the postprocessing stages (PLL/DLL) and allows the continuous accumulation of newly arriving input data.

This first estimate (multiplied by 4 to get the result for the whole receiver) would already utilize $\approx 7 \%$ of the complete FPGA. To reduce complexity, a "curled-up" architecture using the rate difference between processing clock $(100 \mathrm{MHz})$ and data rate (16.8 Msps) was developed and analyzed. This architecture accepts 11 input samples per clock cycle instead of the 51. The timing for this "curled up" architecture is shown in Fig. 6.
Rough calculation of this alternative architecture (cf. Table 7) yielded even worse resource efficiency than the straight forward approach: the accumulator storage still consists of 2 large register banks, additionally Multiplexers and DeMultiplexers are now required to direct the $11 \mathrm{I} / \mathrm{Os}$ to the 51 storage locations.

Further investigations into the "curled-up" architecture were performed to reduce the required register resources (DREGs) and the introduced multiplexers and demultiplexers at the same time. To achieve this goal, the $51 \times 23$ bit accumulator registers had to be mapped onto the dedicated RAM resources available in the Stratix-II FPGA: M512 $(32 \times 18$ bit $)$ and M4K $(128 \times 36$ bit $)$.

This approach requires only very limited additional logic for the adders; the multiplexers and demultiplexers are already available inside the memories addressing capability. For the accumulator storage, a memory depth of 8 was chosen to accommodate for the actually required depth of 5 words. The memories themselves were selected to be "simple dual-ported", therefore read and write accesses can occur at the same time, but not on the same word. All three alternatives have been implemented in VHDL and synthesized. For the RAM-based approach, even an option to force the synthesis to use only the M512 memory resources to increase efficiency in memory utilization was applied. Table 9 shows the results for the Dual Channel Correlation and Discriminators unit, therefore 4 I\&D units were considered.

In conclusion, the Dual Channel Correlation and Discriminators unit is by far the most complex part identified in the GNSS tracking unit. Further optimizations could target an improved memory utilization by sharing memory resources at least over all 4 I\&D units, even better with all I\&D units if multiple receivers are deployed. 
Table 10. Combined implementation results.

\begin{tabular}{lrrr}
\hline Unit & Alternative & ALUT & DREG \\
\hline CWO & Default & $82(1 \%)$ & $76(1 \%)$ \\
Code NCO and PRN gen. & Default & $1172(18 \%)$ & $213(2 \%)$ \\
DCC and Discriminators & Default & $4896(74 \%)$ & $9384(92 \%)$ \\
Residue, Glue Logic, FSM & Estimate & $500(7 \%)$ & $500(7 \%)$ \\
\hline Overall & & 6650 & 10173 \\
\hline
\end{tabular}

\section{Prototype complexity estimation}

The implementation results presented in detail in the previous section are now combined to yield the complexity estimation for the complete dual-channel receiver. As already explained before, residual elements, glue logic and the main FSM are introduced as a lump sum estimate only: 500 ALUTs and 500 DREGs are assumed to cover the required resources even for worst-case scenarios.

The combined implementation results for the stated alternatives (see Table 10 and Fig. 7) add up to 6650 ALUTs and 10173 DREGs which amounts to $\approx 4.6 \%$ and $\approx 7.0 \%$ respectively of one complete Stratix-II FPGA.

In conclusion, up to 14 dual-channel receivers could be theoretically realized on the given FPGA platform incorporating all assumptions and limitations made for this receiver prototyping approach. Because of timing and place and route $(\mathrm{P} \& \mathrm{R})$ limitations, up to 10 full receivers should be feasible. By using the RAM-based approach for the realization of the Dual Channel Correlation and Discriminators unit, the results are not changed significantly: For one dual-channel receiver $7.8 \%$ of the M4K or $12.9 \%$ of the M512 resources of the given FPGA will be required and therefore become the bottleneck. However, if multiple receivers have to be deployed on the same FPGA, the different implementation styles can have massive effects on the scalability of the overall design, especially in terms of timing and P\&R. The implementation complexity of the PLL/DLL as well as the acquisition block and all required internal and external interfaces should also be analyzed to further substantiate these findings.

\section{References}

EUG: European GNSS (Galileo) Open Service Signal In Space Interface Document (OS SIS ICD), European Union, issue 1.1, 2010.

Groh, I. and Sand, S.: Complexity Reduced Multipath Mitigation in GNSS with the Granada Bit-true Software Receiver, in: Proceedings of the IEEE/ION Position Location and Navigation Symposium (PLANS), Monterey, CA, USA, 2008.

Groh, I. and Sand, S.: Optimized Complexity Reduction for Maximum Likelihood Position Estimation in Spread Spectrum Navigation Receivers, to appear in IET Radar, Sonar \& Navigation, 2012.

Groh, I., Staudinger, E., and Sand, S.: Time-Variant Maximum Likelihood Channel Estimation in Mobile Radio Navigation Systems, in: Proceedings of IEEE Vehicular Technology Conference Fall 2011, San Francisco, CA, USA, 2011.

Hu, X., Raasakka, J., Hurskainen, H., Lohan, E.-S., Groh, I., and Sand, S.: Final Report for WP1200, W2200, WP3200, GREAT Project, 2008.

Lentmaier, M. and Krach, B.: Maximum Likelihood Multipath Estimation in Comparison with Conventional Delay Locked Loops, in: Proceedings of ION GNSS, Fort Worth, Texas, USA, 2006.

Selva, J.: Efficient Multipath Mitigation Methods in Navigation Systems, Ph.D. thesis, Universitat Polytecnicia de Catalunya, Barcelona, Spain, 2004.

Selva, J.: An Efficient Newton-Type Method for the Computation of ML Estimators in a Uniform Linear Array, IEEE T. Signal Proces., 53, 2036-2045, 2005.

van Nee, R., Siereveld, J., Fenton, P., and Townsend, B.: The multipath estimating delay lock loop: approaching theoretical accuracy limits, in: Proceedings of the IEEE/ION Position Location and Navigation Symposium (PLANS), 246-251, 1994. 